\title{
Power Efficient Energy-Aware Routing Protocol for Wireless Sensor Networks
}

\author{
Akoijam Premita ${ }^{1}$ Er. Mamta Katiyar ${ }^{2}$ \\ ${ }^{1}$ Department of Electronic and Communication Engineering MMEC of Mullana(Ambala) Hariyana, INDIA \\ ${ }^{2}$ Department of Electronic and Communication Engineering MMEC of Mullana(Ambala) Hariyana, INDIA
}

\begin{abstract}
The most important issue that must be solved in designing a data transmission algorithm for wireless sensor networks (WSNs) is how to save sensor node energy while meeting the needs of applications/users as the sensor nodes are battery limited. While satisfying the energy saving requirement, it is also necessary to achieve the quality of service. In case of emergency work, it is necessary to deliver the data on time. Achieving quality of service in WSNs is also important. In order to achieve this requirement, Powerefficient Energy-Aware routing protocol for wireless sensor networks is proposed that saves the energy by efficiently selecting the energy efficient path in the routing process. When source finds route to destination, it calculates $\alpha$ for each route. The value $\alpha$ is based on largest minimum residual energy of the path and hop count of the path. If a route has higher $\alpha$, then that path is selected for routing the data. The value of a will be higher, if the largest of minimum residual energy of the path is higher and the number of hop count is lower. Once the path is selected, data is transferred along the path. In order to increase the energy efficiency further transmission power of the nodes is also adjusted based on the location of their neighbor. If the neighbors of a node are closely located to that node, then transmission range of the node is decreased. Therefore it is enough for the node to have the transmission power to reach the neighbor within that range. As a result transmission power of the node is reduce which subsequently reduces the energy consumption of the node. Our proposed work is simulated through Network Simulator (NS-2). Existing AODV and Man-Min energy routing protocol also simulated through NS-2 for performance comparison. Packet Delivery Ratio, Energy Consumption and end-to-end delay

(Quality of Service metric) are chosen as performance metrics. Performance of the proposed protocol is compared with the existing AODV and Max-Min energy routing protocol by based on the metrics. Our proposed protocol provides lower energy consumption than AODV and lower delay than the Max-Min energy routing protocol. Thus, our proposed routing protocol saves the energy and also achieves the good quality of service by providing lower delay for data transfer.
\end{abstract}

Keywords: - Power-efficient Energy-Aware routing protocol, AODV, Max-Min energy routing protocol, the largest of minimum residual energy, hop count, Packet Delivery Ratio, Energy Consumption, end-to-end delay

\section{i. Introduction}

A wireless sensor network consists of light-weight, low power, small size of sensor nodes. The areas of applications of sensor networks vary from military, civil, healthcare, and environmental to commercial. Examples of application include forest fire detection, inventory control, energy management, surveillance and reconnaissance, and so on. Due to the low-cost of these nodes, the deployment can be in order of magnitude of thousands to million nodes. The nodes can be deployed either in random fashion or a pre-engineered way. The sensor nodes perform desired measurements, process the measured data and transmit it to a base station, commonly referred to as the sink node, over a wireless channel. The base station collects data from all the nodes, and analyzes this data to draw conclusions about the activity in the area of interest. Sinks can act as gateways to other networks, as a powerful data processor or as access points for human interface. They are often used to disseminate control information or to extract data from the network.

Nodes in sensor networks have restricted storage, computational and energy resources; these restrictions place a limit on the types of deployable routing mechanisms. Additionally, ad hoc routing protocols, for conventional wireless networks support IP style addressing of sources and destinations. They also use intermediate nodes to support end-to-end communication between arbitrary nodes in the network. It is possible for any-to-any communication to be relevant in a sensor network; however this approach may be unsuitable as it could generate unwanted traffic in the network, thus resulting in extra usage of already limited node resources. Many to-one communication paradigms is widely used in regard to sensor networks since sensor nodes send their data to a common sink for processing. This many-to-one paradigm also results in non-uniform energy drainage in the network. Sensor networks can be divided in two classes as event driven and continuous 
dissemination networks according to the periodicity of communication. Routing protocols are usually implemented to support one class of network, in order to increase energy savings. In continuous dissemination networks, routes will be periodically reconstructed, while in event-driven networks routes will be constructed only when an events occurs, since the cost of constant updates is prohibitive in this scenario.

However, sensor nodes are constrained in energy supply and bandwidth. Such constraints combined with a typical deployment of large number of sensor nodes have necessitated energy-awareness at the layers of networking protocol stack including network layer. Routing of sensor data has been one of the challenging areas in wireless sensor network research. Current research on routing in wireless sensor networks mostly focused on protocols that are energy aware to maximize the lifetime of the network, scalable for large number of sensor nodes and tolerant to sensor damage and battery exhaustion. Since the data they deal with is not in large amounts and flow in low rates to the sink, the concepts of latency, throughput and delay were not primary concerns in most of the published work on sensor networks. However, the introduction of imaging sensors has posed additional challenges for routing in sensor networks. Transmission of imaging data requires careful handling in order to ensure that end-to-end delay is within acceptable range. Such performance metrics are usually referred to as quality of service (QoS) of the communication network. Therefore, collecting sensed imaging data requires both energy and QoS aware routing in order to ensure efficient usage of the sensors and effective access to the gathered measurement. QoS protocols in sensor networks have several applications including real time target tracking in battle environments, emergent event triggering in monitoring applications etc.

In this study, Power efficient Energy-Aware Routing Protocol for WSN, which is based upon the ondemand ad hoc routing protocol AODV which determines a proper path with consideration of node residual battery powers. The proposed protocol aims to extend the life time of the overall sensor network by avoiding the unbalanced exhaustion of node battery powers as traffic congestion occurs on specific nodes participating in data transfer. The remainder of the paper is organized as follow. Section II deals with related work done on the area of wireless sensor networks in routing. It discusses the various types of routing and the core idea of each kind. Section III discusses the proposed methodology, its architecture, block diagram and description of each module to be implemented in the simulation. Section IV performance of the result comparison of three protocols and analyses the performance of proposed protocol against the existing AODV and Max_Min energy protocol. Section V provides the conclusion of the work and future scope.

\section{ii. $\quad$ Related Works}

There are four main categories of routing protocols in WSN. They are data-centric, hierarchical, location-based [4] and multipath:

In data-centric routing, the sink sends queries to certain regions and waits for data from the sensors located in the selected regions. Since data is being requested through queries, attribute based naming is necessary to specify the properties of data. Five of the main algorithms are SPIN [15] (meta-data negotiation solves the classic problems of flooding such as redundant information passing, overlapping of sensing areas and resource blindness thus, achieving a lot of energy efficiency), Directed Diffusion [13] [16] (each node disseminate the date interest in receive), Rumor routing [29] is another variation of Directed Diffusion and is mainly intended for contexts in which geographic routing criteria are not applicable. Gradient-Based Routing (The difference between a node's height and that of its neighbor is considered the gradient on that link. A packet is forwarded on a link with the largest gradient [6]) and constrained anisotropic diffusion routing (CADR) is a protocol [7], which strives to be a general form of Directed Diffusion. Hierarchical algorithms separate the node in sub-regions called cluster in order to segregate the areas of monitoring environment as LEACH, PEGASIS \& Hierarchical PEGASIS [35] and TEEN \& APTEEN [9]. The main aim of hierarchical routing is to efficiently maintain the energy consumption of sensor nodes by involving them in multi-hop communication within a particular cluster and by performing data aggregation and fusion in order to decrease the number of transmitted messages to the sink. Cluster formation is typically based on the energy reserve of sensors and sensor's proximity to the cluster head [18] [19]. Location-Based algorithms (i.e. MECN \& SMECN [17] and GAF [10]) rely on the use of routing protocols for sensor networks require location information for sensor nodes. In most cases location information is needed in order to calculate the distance between two particular nodes so that energy consumption can be estimated. Since, there is no addressing scheme for sensor networks like IPaddresses and they are spatially deployed on a region, location information can be utilized in routing data in an energy efficient way. Finally, Multipath algorithms uses of multipath routing protocols are based on classic ondemand single path routing methods [11] [12], such as AODV and DSR. They differ from each other on how to forward multiple route requests and how to select multiple routes. In some papers, node energy is also taken into account when constructing multiple paths [5] (i.e. EECA [2]). 


\section{Energy Aware Routing}

EAP, the operation of EAP is divided into rounds as LEACH. Each round begins with a set-up phase while clusters are organized and the routing tree is constructed, followed by a working phase when data are sent to the sink node [3].

\section{Power Aware Routing}

\section{Real-time Power-Aware Routing}

Real-time Power-Aware Routing in Sensor Networks Due to the unreliable and dynamic nature of WSNs, it is unrealistic to provide hard delay guarantees. RPAR focuses on minimizing the energy consumed in packet transmissions. In addition, RPAR is designed based on the following principles:

- WSN applications have varied communication requirements resulting in workloads with diverse deadlines. A real-time power-aware routing protocol should dynamically adapt its transmission power and routing decisions based on workload and packet deadlines.

- The design of RPAR should account for the realistic characteristics of WSNs including loss links and extreme resource constraints in terms of memory, bandwidth and energy.

- RPAR should be localized protocol that makes decisions based solely on one-hop neighborhood information. This property enables RPAR to scale effectively to large WSNs.

\section{Power aware algorithm \\ Max-min z P min algorithm}

requires information about the power level of each computer in the network. Having accurate knowledge of this information is not a problem in small networks. However, for large networks it is difficult to aggregate and maintain this information. This makes it hard to implement the max-min zPmin algorithm for large networks.

\section{Zone-based routing}

is a hierarchical approach in which the area covered by the (sensor) network is divided into a small number of zones. Each zone has many nodes and thus there is a lot of redundancy in routing a message through it. To send a message across the entire area we find a 'global' path from zone to zone and give each zone control over how to route the message within itself. Thus, zone-based power-aware routing consists of (i) an algorithm for estimating the power level of each zone; (ii) an algorithm for computing a path for each message across zones; and (iii) an algorithm for computing the best path for the message within each zone (with respect to the power lifetime of the zone.).

\section{Existing System}

\section{iii. Proposed Methodology}

AODV is a typical routing protocol for MANETs. When a node wants to find a route to another one it broadcasts a RREQ to the entire network till either the destination is reached or another node is found with a fresh enough route to the destination. Then a RREP is sent back to the source and the discovered route is made available. Nodes that are part of an active route may offer connectivity information by broadcasting periodically local Hello messages (special RREP messages) to its neighbors. If Hello messages stop arriving from a neighbor beyond some time threshold, the connection is assumed to be lost. When a node detects that a route to a neighbor node is not valid it removes the routing entry and sends a RERR message to neighbors that are active and use the route; this possible by maintaining active neighbors lists. This procedure is repeated at nodes that receive RERR messages. A source that receives an RERR can reinitiate a RREQ message. This routing process will not consider about the energy of the node and it only considers the hop-count along the path.

Max Min energy routing protocol chooses the route with largest minimum residual energy. It does not consider the hop count along the path.

\section{Proposed System}

In the proposed system we focus on the problem of maximizing the lifetime of a wireless sensor network where the sensor nodes communicate with the sink by delivering the sensed data across multiple hops with different transmission energy requirements. That is, there is flexibility of transmitter power adjustment and the energy consumption rate per unit information transmission is not the same for all neighbors of a sensor, but depends on the choice of the next hop node. The lifetime of the network is defined as the time until a sensor 
node drains out of battery energy for the first time. Proposed system implements the energy saving routing protocol in the battery limited wireless sensor network in order the lifetime of the network. The proposed protocol performs a route discovery process similar to the AODV protocol. But it considers the residual energy level of the node and hop count along the path towards the sink. (Minimum Residual Energy) field is added to the RREQ message. The Min-RE field is set as a default value of -1 when a source node broadcasts a new RREQ message for a route discovery process. To find a route to a destination node, a source node floods a RREQ packet to the network. When neighbor nodes receive the RREQ packet and update the Min-RE value and rebroadcast the packet to the next nodes until the packet arrives at a destination node. That is, the proposed protocol collects routes that have the minimum residual energy of nodes relatively large and have the least hopcount, and then determines a proper route among them, which consumes the minimum network energy compared to any other routes. It uses the formula to select the optimum route. The formula is based on the hop count and the Minimum Residual Energy.

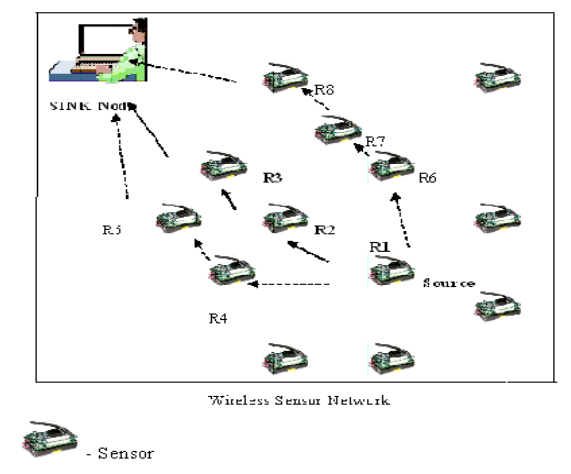

Figure1: Architecture of WSNs Pouting

\section{Block Diagram:}

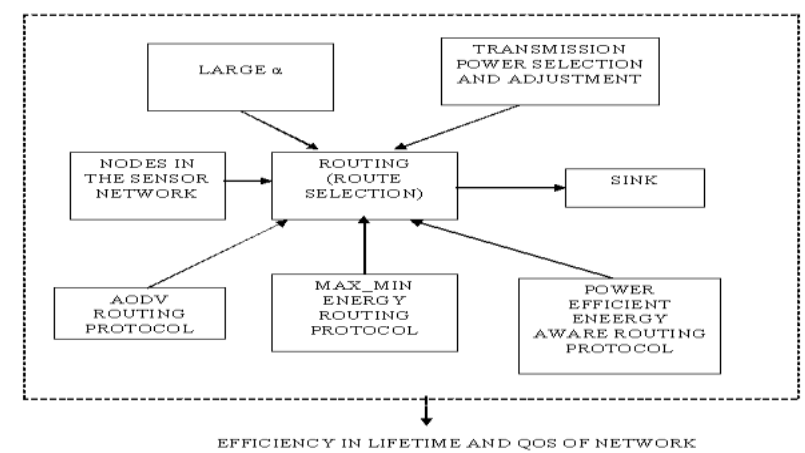

Figure2: Block Diagram

$\alpha=$ Min-RE / No. Of hops

(1)

\subsection{Modules Description}

Proposed routing protocol

MODULE 1: Wireless Sensor Network setup

MODULE 2: Function of the Routing Protocol

MODULE 3: Finding the Optimum route based on $\alpha$

MODULE 4: Energy saving Protocol

MODULE 5: Analysis of routing protocols

\section{MODULE 1}

Wireless Sensor Network setup

This work considers that $\mathrm{N}$ sensor nodes are scattered in a two-dimensional square field $\mathrm{A}$, and the sensor network has the following properties: This network is a static densely deployed network. It means a large number of sensor nodes are densely deployed in a two-dimensional geographic space, forming a network. There exists only one sink, which is deployed at a fixed place outside A. 
For a sensor node, there are three kinds of methods to get its location information i.e. GPS, directional antenna and position algorithms. However, obtaining location information with a hardware device such as GPS or directional antennae will cause an increase in the cost of a sensor node, and position algorithms that need to exchange a large quantity of messages to compute the node's location information will also result in high energy consumption. Nodes will exchange about their location information and residual energy level in the routing process. In this way, the node energy consumption can be remarkably reduced so as to further prolong sensor network lifetime.

\section{MODULE 2}

\section{Function of the Routing Protocol}

The proposed protocol performs a route discovery process by considering the network lifetime and performance; that is, considering residual energy of nodes on the path and hop count. In order to implement such functions, a new field, called Min-RE (Minimum Residual Energy) field, is added to the RREQ message. The Min-RE field is set as a default value of -1 when a source node broadcasts a new RREQ message for a route discovery process.

\begin{tabular}{|c|c|c|c|c|c|c|c|}
\hline Type & $\mathrm{J}$ & $\mathrm{R}$ & $\mathrm{G}$ & $\mathrm{D}$ & $\mathrm{U}$ & Reserved & $\begin{array}{c}\text { Hop } \\
\text { Count }\end{array}$ \\
\hline \multicolumn{3}{|c|}{ RREQ ID. } \\
\hline \multicolumn{3}{|c|}{ Destination IP Address } \\
\hline Destination Sequence Number \\
Originator IP Address \\
\hline Originator Sequence Number \\
\hline Min-RE(Added)
\end{tabular}

Figure3: A RREQ message format for our Proposed protocol

To find a route to a destination node, a source node floods a RREQ packet to the network. When neighbor nodes receive the RREQ packet and update the Min-RE value and rebroadcast the packet to the next nodes until the packet arrives at a destination node. If the intermediate node receives a RREQ message, it increases the hop count by one and replaces the value of the Min-RE field with the minimum energy value of the route. In other words, Min-RE is the energy value of the node if Min-RE is greater than its own energy value; otherwise Min-RE is unchanged. If the destination node finally receives the first RREQ message, it triggers the data collection timer and receives all RREQ messages forwarded through other routes until time expires.

\section{MODULE 3}

\section{Finding the Optimum route based on}

$\alpha$ :The optimum route is determined by using the value of $\alpha$ described in formula (1). The destination node calculates the values of $\alpha$ for received all route information and choose a route that has the largest value of $\alpha$. That is, the proposed protocol collects routes that have the minimum residual energy of nodes relatively large and have the least hop-count, and then determines a proper route among them, which consumes the minimum network energy compared to any other routes.

$\alpha=$ Min-RE / No. Of hops

Here Min-RE is the minimum residual energy on the route and No-Hops is the hop count of the route between source and destination.

\section{MODULE 4}

\section{Energy Saving Protocol}

As the senor nodes are energy constraint nodes, they must take action to save their energy as far as possible. Transmission range adjustment protocol is also executed so that to conserve energy along with the routing process. Two phases are there. 1 . Transmission range selection phase 2 . Transmission range adjustment phase. At start of the routing process, transmission range selection phase is executed. After route finding, transmission range adjustment phase is executed. 


\section{i) Transmission range selection phase:}

Higher transmission power will give higher range of communication range. Normally nodes will have the transmission power that provides the coverage to the distance that is much higher than the distance between the node and its extreme neighbor. This leads to the energy wastage which is the main constraint for sensor nodes to operate for longtime. Initially a node will send hello packets to its neighbor and collect the information from the neighbor then find out the distance from it to its neighbors. Then it selects the maximum distance from the calculated distance and set that distance as initial the transmission range.

\section{ii) Transmission range adjustment phase:}

After some time of communication, energy will be reduced in each node. If the node still uses the initial transmission range, its energy will be completely worn out and it cannot involve in further communication. In order to prevent the node from that, transmission range readjustment phase is executed in which nodes transmission power is reduced. This will lead to i) reduction of transmission range (coverage) ii) Reduction of energy consumption iii) reduction of number of neighbor nodes iv) increase the lifetime of the node in the network. So transmission range must be adjusted based on coverage index required and also based on the remaining energy, duration communication it will involve further.

\section{MODULE 5}

\section{Analysis of routing protocols}

To understand the operations of the proposed protocol, it considers three different routing protocols for operational comparison:

Case 1: Choose a route with the minimum hop count between source and destination (AODV routing protocol). Case 2: Choose a route with largest minimum residual energy. (Max_Min Energy (Min-ER) routing protocol) Case 3: Choose a route with the large minimum residual energy and less hop count i.e. with the longest network lifetime (proposed routing protocol).

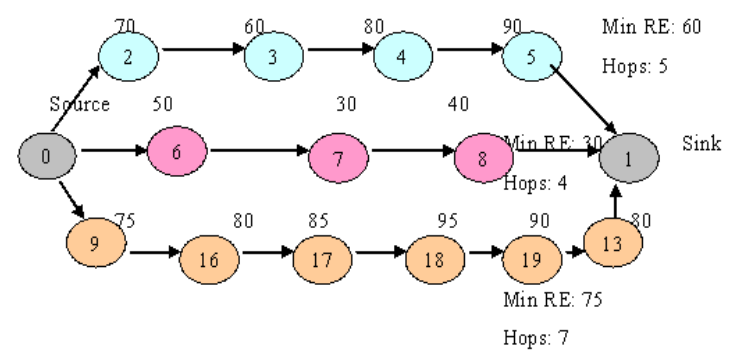

Figure4: A sample network for establishment a routing path

Case 1 considers only the minimum hop count, it selects route <0-6-7-8-1> which has the hop count of 4 . In the Case-2, select route <0-9-16-17-18-19-13-1> which has Min-RE 75 is chosen because the route has the largest minimum residual energy among routes. In this study, proposed model needs to compute the value of $\alpha$ by using formula (1), and selects a route with largest value of $\alpha$. Thus Case 3 selects route $<0-2-3-4-5-1\rangle$ which has largest $\alpha$ value of 12. Case 1 selects the shortest path without considering residual energy of nodes, which is the same as the AODV routing algorithm. This case does not sustain a long lifetime in the network. Case 2 selects a route with largest minimum residual energy to extend network lifetime but it has serious problem in terms of the hop count. Case- 3 improves the drawbacks of Case 1 and Case- 2 by considering both residual energy and hop count. It extends network lifetime by arranging almost all nodes to involve in data transfer. The proposed protocol also selects a route with the longest lifetime in the network without performance degradation such as delay time and node energy consumption. Packet delivery ratio, delay and energy consumption are considered as performance metrics. These performance metrics are calculated for the existing AODV, Max_Min Energy (MinER) routing protocol) and proposed routing protocol. Packet delivery ratio is similar to both the existing and proposed protocols. But proposed routing protocol achieves minimum energy consumption, and lesser delay than the existing protocols. Thus proposed routing protocol increases the lifetime of the node in the network and also increases the Quality of Service of the communication network. 


\section{iv. Performance Analysis}

Ns2 is a simulation tool built by South-California University and regenerated by ISI and some others. The NS2 was built using three languages. TCL script, C++, C. Here, TCL used for control, C++ for data and most of the header files were created by C. In NS2 Scripting, we can simulate a wired, wireless and Satellite networks using ns script. And the ns scripted files are saved with the extension of *.tcl. (TCL: Tool Command Language).

\subsection{Simulation Mode}

An extensive simulation model having scenario of $\mathrm{n}$ mobile nodes and $\mathrm{n}$ UDP/TCP connections is used to study inter-layer interactions and their performance implications.

The other parameters used in this model are as under:

Table1: Simulation model specification

\begin{tabular}{|l|l|}
\hline Software for simulation & Network simulator 2. \\
\hline Channel & wireless \\
\hline Simulation runs time & 30 seconds \\
\hline Area in which nodes move & $600 \mathrm{X} 600$ \\
\hline Packet size & 1024 bytes \\
\hline Speed & $1 \mathrm{~m} / \mathrm{s}$ to $10 \mathrm{~m} / \mathrm{s}$ \\
\hline Routing Protocol & AODV \\
\hline Propagation model & TwoRay Ground \\
\hline Network Interface Type & Wireless Physical \\
\hline Queue Type & Drop Tail \\
\hline IFQ-Length & 50 Packets \\
\hline MAC Type & Mac/802.11 \\
\hline Antenna Type & Omni Antenna \\
\hline
\end{tabular}

Network simulator NS2 is used for the simulation of the scenario. Totally 20 nodes are used in the simulation and they are placed in the area of 600X600. Wireless channel is used in the simulation process. Drop Tail queue is used. Mac/802.11 is used and omni directional antenna is used. Signal propagation uses Two Ray Ground model. UDP is used as transport agent. CBR is used for traffic generation with rate of 1024 bytes per second. The performance is analyzed over the wireless sensor network using generated packets, received packets, dropped packets, packet delivery ratio, delay, energy consumption and coverage.

\subsection{Performance Metrics Packet Delivery Ratio}

PDR is the proportion to the total amount of packets reached the receiver and amount of packet sent by source. If the amount of malicious node increases, PDR decreases. The higher mobility of nodes causes PDR to decrease.

$\operatorname{PDR}(\%)=$ Number of packets successfully delivered to destination

Number of packets generated by source node

\section{End-to-End Delay}

End-to-End delay is the time taken for a packet to reach the destination from the source node. 
Total number of delivered data packets

\section{Energy Consumption}

Energy consumption of the network is the sum of energy consumption of all the nodes in the network. Energy consumption each node is the difference between the energy of the node at the start of the communication and the energy of the node at the end of the communication.

Energy consumption (Joules) $=\sum$ (Initial energy - Residual Energy)

Table2: Performance metrics comparison among three protocols

\begin{tabular}{|l|l|l|l|}
\hline Routing Protocol & $\begin{array}{l}\text { Energy } \\
\text { Consumption }\end{array}$ & Delay & Packet Delivery Ratio \\
\hline AODV & Higher (263.209) & - & $99.8707 \%$ \\
\hline Max_Min Energy & - & Higher (0.79961) & $95.8867 \%$ \\
\hline Proposed & Lower (159.041) & Lower(0.37708) & $99.7931 \%$ \\
\hline
\end{tabular}

In table 1 performance metrics comparison among three protocols have been done. Delay must be compared between Max_Min Energy and Proposed protocol. Because both protocol's operation varies only in the hop count. Hop count is directly proportional to delay. Energy is not compared because both protocols select the higher residual energy path.

Energy must be compared between AODV and Proposed protocol. Because both protocol's operation varies only in energy and transmission power adjustment consideration. Energy consumption is directly proportional to the transmission power of the node. Hop is not compared because both protocols select the less hop count path.

\subsection{Simulation Results}

The Packet Delivery Ratio of all three routing protocols provides good Packet delivery in the range of 95-100\%. But in Max-Min Energy routing protocol give fluctuation. Fluctuations in the packet delivery ratio are due to the many hops in the routing path.

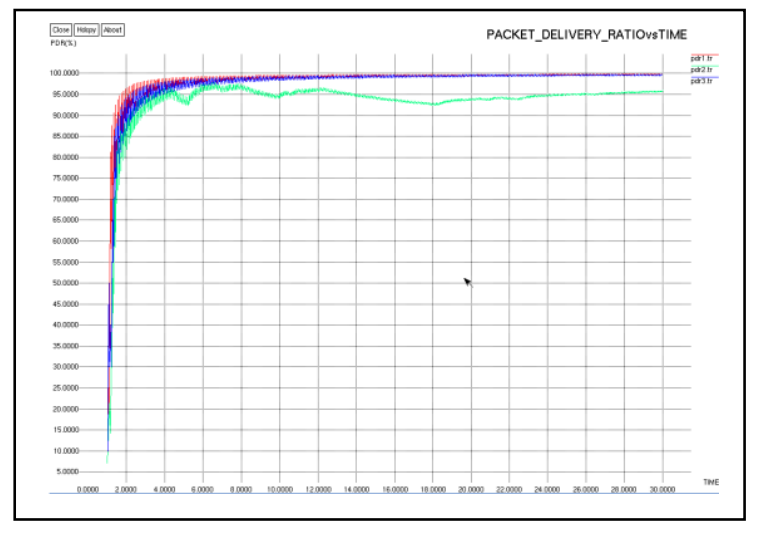

Figure 5: Packet Delivery Ratio versus Time (Three Routing protocols)

In Figure5 Red line represents the result of AODV Blue line represents the result of Proposed Protocol. Green line represents the result of Max_Min Energy routing protocol. Packet Delivery ratio is almost similar to all the protocols. But Max_Min energy routing protocol produces the less packet delivery ratio. 


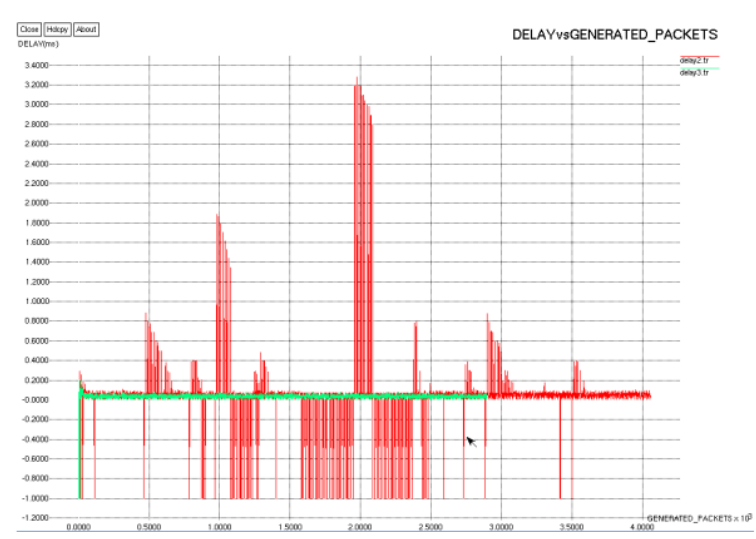

Figure 6: Delay versus Generated Packets (Proposed and Max_Min routing protocol)

In Figure6 Red line represents the result of Max_Min Energy routing protocol Max_Min Energy routing protocol Green line represents the result of Proposed Protocol. Proposed routing protocol provides lesser delay than the Max_Min energy routing protocol.

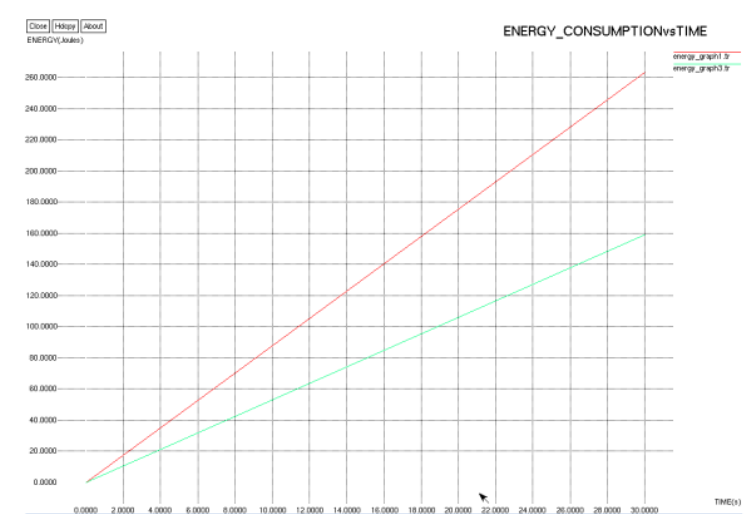

Figure7: Energy Consumption versus Time (Proposed and AODV Routing protocols)

In Figure7 Red line represents the result of AODV routing protocol. Green line represents the result of Proposed Protocol. Proposed routing protocol provides lesser energy consumption than the AODV routing protocol.

\section{Proposed routing protocol provides lesser energy consumption than the AODV routing protocol.}

Reason:

Proposed routing protocol allows the higher energy routing path and also adjusts the transmission power based on the transmission range. But AODV does not consider the energy of the nodes in the routing path and the transmission range and power.

\section{Proposed routing protocol provides lesser delay than the Max_Min energy routing protocol.}

Reason:

Max_Min energy routing protocol does not consider the hop count. Therefore there may be the implementation of higher hop count path for routing. Higher hop count leads to higher delay. But proposed routing protocol considers the hop count as a measure for routing. Hop count must be less for the proposed protocol. Less hop count leads to less delay.

Packet Delivery ratio is almost similar to all the protocols. But Max_Min energy routing protocol produces the less packet delivery ratio.

Reason:

Max_Min energy routing protocol does not consider the hop count. Higher hop count path leads to decrement in packet delivery ratio. 


\section{v. Conclusion and Future Scope}

Proposed Energy efficient routing protocol for wireless sensor network invokes the residual energy and hop count as parameters. In the routing process path with largest minimum residual energy and least hop count is chosen. Transmission power of the node is adjusted according to neighbor's range of the node. Proposed Energy efficient routing protocol is compared with the existing protocols. Proposed protocol achieves the higher energy consumption. This improves the lifetime of the nodes in the network. Quality of Service of the communication network is also improved by achieving the lesser end-to-end delay. Thus proposed routing protocol provides better lifetime and Quality of Service than the AODV and Max_Min energy routing protocol. In the future scope new routing algorithms are needed in order to handle the overhead of mobility and topology changes in such energy constrained environment Other possible future research for routing protocols includes the integration of sensor networks with wired networks (i.e. Internet). Most of the applications in security and environmental monitoring require the data collected from the sensor nodes to be transmitted to a server so that further analysis can be done. On the other hand, the requests from the user should be made to the sink through Internet. Since the routing requirements of each environment are different, further research is necessary for handling these kinds of situations.

[1] Adeel Akhtar, Abid Ali Minhas, and Sohail Jabbar, "Energy Aware Intra Cluster Routing for Wireless Sensor Networks", International Journal of Hybrid Information Technology Vol.3, No.1, January, 2010

[2] Zijian Wang, Eyuphan Bulut, and Boleslaw K. Szymanski, "Energy Efficient Collision Aware Multipath Routing for Wireless Sensor Networks", International Conference on Communication June 14-18, 2009.

[3] An Energy-Aware Routing Protocol in Wireless Sensor Networks Ming Liu 1, Jiannong Cao 2, Guihai Chen 3 and Xiaomin Wang Sensors 2009

[4] K. Akkaya, and M. Younis, "A Survey on Routing Protocols for Wireless Sensor Networks", Elsevier Ad Hoc Network Journal, vol. 3, no. 3, pp 325-349, 2005.

[5] A.P. Subramanian, A.J. Anto, J. Vasudevan, and P. Narayanasamy, "Multipath power sensitive routing protocol for mobile ad hoc networks", Proc. Conf. Wireless on Demand Network Systems, 2004, LNCS 2928, 2004, pp. 171-183.

[6] C. Schurgers and M.B. Srivastava, "Energy efficient routing in wireless sensor networks," in the MILCOM Proceedings on Communications for Network-Centric Operations: Creating the Information Force, McLean, VA, 2001.of the First Workshop on Sensor Networks and Applications (WSNA), Atlanta, GA, October 2002.

[7] M. Chu, H. Haussecker, and F. Zhao, "Scalable Information-Driven Sensor Querying and Routing for ad hoc Heterogeneous Sensor Networks," The International Journal of High Performance Computing Applications, Vol. 16, No. 3, August 2002.

[8] S. Lindsey and C. S. Raghavendra, "PEGASIS: Power Efficient GAthering in Sensor Information Systems," in the Proceedings of the IEEE Aerospace Conference, Big Sky, Montana, March 2002.

[9] A. Manjeshwar and D. P. Agrawal, "APTEEN: A Hybrid Protocol for Efficient Routing and Comprehensive Information Retrieval in Wireless Sensor Networks", in the Proceedings of the $2^{\text {nd }}$ International Workshop on Parallel and Distributed Computing Issues in Wireless Networks and Mobile computing, Ft. Lauderdale, FL, April 2002.

[10] Y. Xu, J. Heidemann, and D. Estrin, "Geography-informed energy conservation for ad hoc routing," in the Proceedings of the 7th Annual ACM/IEEE International Conference on Mobile Computing and Networking (MobiCom'01), Rome, Italy, July 2001.

[11] M.K. Marina, and S.R. Das, "On-demand multipath distance vector routing in ad hoc networks," Proc. 9th IEEE Int. Conf. Network Protocols (ICNP), 2001, pp. 14-23.

[12] S.J. Lee and M. Gerla, "Split multipath routing with maximally disjoint paths in ad hoc networks," Proc. IEEE Int. Con. Communications (ICC),2001, vol.10, pp. 3201-3205.

[13] C. Intanagonwiwat, R. Govindan and D. Estrin, "Directed diffusion: A scalable and robust communication paradigm for sensor networks", in the Proceedings of the 6th Annual ACM/IEEE International Conference on Mobile Computing and Networking (MobiCom'00), Boston, MA, August 2000.

[14] Charles E. Perkins and Elizabeth M. Royer. "Ad hoc On-demand Distance Vector Routing." Proceedings of the 2nd IEEE Workshop on Mobile Computing Systems and Applications, New Orleans, LA, pp. 90-100, February 1999.

[15] W. Heinzelman, J. Kulik, and H. Balakrishnan, "Adaptive protocols for information dissemination in wireless sensor networks," in the Proceedings of the 5th Annual ACM/IEEE International Conference on Mobile Computing and Networking (MobiCom'99), Seattle, WA, August 1999.

[16] D. Estrin, et al., "Next century challenges: Scalable Coordination in Sensor Networks," in the Proceedings of the 5th annual ACM/IEEE international conference on Mobile Computing and Networking (MobiCom'99), Seattle, WA, August 1999.

[17] V. Rodoplu and T.H. Ming, "Minimum energy mobile wireless networks," IEEE mJournal of Selected Areas in Communications, Vol. 17, No. 8, pp. 1333-1344, 1999.

[18] A. Buczak and V. Jamalabad, "Self-organization of a Heterogeneous Sensor Network by Genetic Algorithms," Intelligent Engineering Systems Through Artificial Neural Networks, C.H. Dagli, et. (eds.), Vol. 8, pp. 259-264, ASME Press, New York, 1998.

[19] C.R. Lin and M. Gerla, "Adaptive Clustering for Mobile Wireless Networks", IEEE Journal on Selected areas in Communications, Vol. 15, No. 7, September 1997.

[20] Information Sciences Institute, "The Network Simulator ns-2" http://www.isi.edu/nanam/ns/, University of Southern California.

[21] http://www.omnetpp.org

[22] http://www.j-sim.org

[23] http://www.cs.rpi.edu/ cheng3/sense 